\title{
Estado de la cuestión y nuevas perspectivas en la evaluación de la calidad percibida en los cibermedios
}

\author{
Francisco HERnÁNDEZ SORIANO \\ Universidad de Lleida \\ paco.hernandez@udl.cat \\ Eduard CRISTÓBAL Fransi \\ Universidad de Lleida \\ ecristobal@aegern.udl.cat
}

\begin{abstract}
Resumen
Los cibermedios, debido al medio en el que se desenvuelven, están en continua expansión y evolución, de modo que es aconsejable para las empresas que participan en los mismos disponer de unos sistemas de evaluación de la calidad percibida acorde a dicha evolución. Para ello, es imprescindible complementar los métodos básicos de medición de la calidad percibida con aquellos parámetros que puedan conseguir una ventaja competitiva sobre el resto de portales de información.
\end{abstract}

Palabras clave: cibermedios; calidad percibida; comunicación 2.0; periodismo digital

\section{State of the art and new perspectives in the evaluation of the perceived service quality in online media}

\begin{abstract}
The online media due to the environment, in which they operate, are constantly expanding and evolving, so it is advisable for companies that participate in the same assessment systems perceived quality according to these developments. Therefore, it is essential to complement the basic methods for measuring the perceived service quality with parameters that can gain a competitive advantage over other online media.
\end{abstract}

Keywords: online media; perceived quality; web 2.0; online journalism

\section{Referencia normalizada:}

Hernández Soriano, F.; Cristóbal Fransi, E. (2013) Estado de la Cuestión y nuevas perspectivas en la evaluación de la calidad percibida en los cibermedios. Historia y Comunicación Social. Vol. $18 \mathrm{~N}^{\mathrm{o}}$ Especial Noviembre. Págs. 15-27.

Sumario: 1. Introducción. 2. Dimensión A-Eficiencia. 3. Dimensión B-Disponibilidad del Sistema. 4. Dimensión C-Fiabilidad y Privacidad. 5. Dimensión D-Interacción. 6. Conclusiones. 7. Bibliografía. 


\section{Introducción}

Son los propios creadores de la escala de medición de la calidad percibida, E-S-QUAL, quienes reconocen que "...no ha sido aplicada a otros tipos de sitios web que no tenga por objeto las ventas online", y que "se han de realizar las adaptaciones necesarias y evaluar las propiedades psicométricas de dichas escalas adaptadas" (Parasuraman et al., 2005. Pág. 214). Del mismo modo, otros autores también relacionados con el estudio de la capacidad percibida, determinaban que: "Una página web que no cuida su diseño e interacción con el internauta proyecta una imagen pobre en Internet y pierde competitividad" (Barnes et al., 2002. Pág. 114). Es por ello que, en base a una revisión de la literatura existente hasta la fecha, hemos desarrollado un instrumento de medición de la calidad percibida en los portales de información que incluye, además de las cuatro dimensiones que propone la escala E-S-QUAL, una más concerniente a la Interacción con el internauta.

\section{Dimensión A-Eficiencia}

En el análisis del modelo E-S-QUAL realizado por Petnji et al. (2012), que examina los estudios realizados en once países diferentes, la dimensión Eficiencia aparece en veinte de los veinte estudios examinados como una de las dimensiones finales a tener en cuenta de cara a la calidad percibida.

\subsection{Usabilidad (a.1)}

Autores como Barnes y Vidgen $(2002,2003)$ nos presentan, mediante sus diversos estudios, que la usabilidad es uno de los pilares sobre los que se puede ofrecer al cliente un mejor servicio y ganar un mayor posicionamiento respecto al resto de empresas. Conclusión a la que también llegan Cox y Dale (2002). Del mismo modo, Palacios y Noci (2008) también confieren una gran importancia a este ítem puesto que se trata de comprobar "...el nivel de adecuación de sus contenidos a los usuarios con características especiales... o que utilicen tecnologías de capacidad limitada (como agendas electrónicas y teléfonos móviles)" (Rodríguez et al., 2010. Pág. 37)

\subsection{Web 2.0 (a.2)}

Macià (2007) recalca que la Web 2.0 nos facilita el contacto a través de nuestra página web con blogueros, prescriptores, analistas, importadores, exportadores, periodistas, etc... Coincide Gosende (2011), en cuanto al hecho de usar la Web 2.0 como una herramienta para mejorar el posicionamiento en buscadores de nuestro sitio Web. También Rodríguez y Pedraza (2009) concluyen en su estudio que las herramientas Web 2.0 suponen un cambio en la forma de concebir el proceso de comunicación con los usuarios, de manera que se puedan utilizar para difundir nuestros contenidos más allá de nuestro propio sitio web. 
2.3 Acceso al portal de información a través de plataformas físicas y digitales (a.3)

La importancia de las redes sociales radica en que “...de esta manera la noticia se traslada desde un contexto puramente periodístico, como es el diario en línea, a un contexto social donde el medio actúa como un usuario más" (Rodríguez Martínez et al., 2010. Pág. 42). Asimismo, Lee et al. (2007) revelaron mediante su investigación que una de las maneras más cómodas de acceder a una web, sin conocer su enlace directo, era a través de sistemas de búsqueda. De ahí que, tal y como indicaban Rodríguez y Pedraza (2009), haya habido una mejora en los motores de búsqueda, de modo que los internautas tengan, cada vez más, acceso a toda aquella información que desean explorar.

\subsection{Recursos audiovisuales o multimedialidad (a.4)}

Según Parra et al. (2008), los Cibermedios tienen ventaja sobre el formato papel al poder complementar la noticia con contenidos multimedia. Y más, Codina (2000), Kawamoto (2003), así como Salaverría y Noci (2003), Masip et al. (2010) destacan la multimedialidad como una de las propiedades básicas que ha de tener la información on-line de cara al usuario. Aunque fue en un trabajo realizado por Salaverría et al. (2005) donde se ideó una propuesta de tipificación de Cibermedios en base al "grado de adecuación de éstos", siendo la multimedialidad uno de ellos.

\subsection{Profundización de la información (a.5)}

Vázquez-Casielles et al. (2009) en su trabajo sobre las agencias de viaje virtuales abordaron, no sólo si la información ofrecida era relevante, sino también otros aspectos como si la información sobre la que se trataba era lo suficientemente amplia y detallada. Así mismo, Cristóbal y Marimon (2010. Pág. 96) determinan que: “a nivel de consumidor, una de las principales ventajas es el acceso a una gran cantidad de información que le permite tener más posibilidades de elección sin tener en cuenta la localización geográfica". Del mismo modo, Rodríguez-Martínez et al. (2010), al igual que López y Pastor (2010), destacan, entre los servicios novedosos que ofrecen los Cibermedios, el de la nube de términos en los blogs.

\subsection{Actualización de la información (a.6)}

Dentro de los indicadores que comentábamos anteriormente en el trabajo de Rodríguez et al. (2010), la actualización de la web forma parte del indicador relacionado con la profundización de la información: ¿Aparece la hora de la última actualización? Y es que, tal y como determinan Salverría et al. (2005), una de las cualidades del periodismo virtual es la inmediatez con la que estos se puedan actualizar. De igual forma recalcan tal hecho otros autores como Perceval (2008), Scolari et al. (2008), y Parra y Álvarez (2004), Cristóbal y Marimon (2011), y Vila López et al. (2013), recalcando que la actualización de la información y los servicios web son esenciales a la hora de hablar del posicionamiento web de un producto. 


\title{
3. Dimensión b - disponibilidad del sistema
}

\begin{abstract}
"No es suficiente un sistema de navegación para garantizar el acceso a la información que contiene una web, sino que, en realidad, y salvo excepciones muy determinadas, cualquier sede web medianamente compleja requerirá de un sistema de recuperación para complementar el sistema de navegación si se desea garantizar el acceso a la información" (Codina, 2000. Pág. 19).
\end{abstract}

\subsection{Agilidad de la web (b.1)}

Basándonos en estudios previos de Lin y Lu (2000) podemos deducir que existe una relación entre la velocidad de interacción y el tiempo de respuesta cuando el internauta navega y su actitud general hacia la web. Algo que también concluyó Rodríguez (2000), quien indicaba que era importante tener en cuenta la velocidad de navegación y el ancho de banda a la hora de establecerse en la Red.

\subsection{Necesita aplicaciones (b.2)}

Un website debe estar preparado para que, no sólo el portal de información pueda emplear sus propios recursos, sino que el internauta no tenga dificultades a la hora de utilizarlos, según Rodríguez y Pedraza (2009). De la misma opinión es Cebrián (2010) que hace hincapié en los Cibermedios como un puente de interacción entre el usuario, la información y el diario digital: “...los equipos que deben emplearse van más allá de lo instrumental para erigirse en mediadores que conforman los procesos comunicativos y establecen diversos sistemas de control mediante los diseñadores de software, las empresas operadoras y los servidores, además de las mediaciones del propio cibermedio” (Cebrián, 2010. Pág. 19)

\subsection{Necesita el uso de conocimientos más complejos (b.3)}

En un estudio llevado a cabo por Pavlou (2003) éste concluye que la facilidad de uso tiene un efecto significativo sobre el uso de transacciones de tipo electrónico ya que reduce la incertidumbre del usuario a la hora de utilizar al servicio. Por otro lado, Sánchez y Villarejo (2004), en su análisis de los efectos moderadores del comportamiento de uso de la web, concluyen que: "la facilidad de uso influye positivamente en la calidad del servicio electrónico” (Sánchez y Villarejo, 2004. Pág. 123)

\subsection{Sistema de búsqueda (b.4)}

Son Wolfinbanger y Gilly (2003) quienes logran determinar cuatro factores imprescindibles a la hora de estudiar la calidad percibida, entre los cuales se encuentra el hecho de que exista un sistema de búsqueda que ayude al cliente y mejore, de este modo, su experiencia en referencia a la página web visitada. Del mismo modo, Rodríguez y Pedraza (2010) usan dichas plataformas digitales como un indicador específico, al tiempo que López y Pastor (2010) nos las presentan como sistemas 
correspondientes a un Cibermedio avanzado. También Cabrera (2010) destaca este apartado como uno de los indicadores más fiables a la hora de determinar si una página web ha evolucionado y se ha adaptado a las nuevas tecnologías.

\subsection{Sistema de ayuda y mapa web (b.5)}

Jakob Nielsen (2008) confirmaba que los mapas de la propia web continuaban siendo útiles como sistemas de navegación secundarios. Por otro lado, Rodríguez et al. (2010 y 2012) determinaban los puntos de acceso a la información como uno de los indicadores generales de la calidad de los contenidos en línea: búsqueda simple, búsqueda avanzada, hemeroteca y mapa del sitio web.

3.6 Servicio de información o compra disponible todo en un día (b.6)

Son Pérez y Perea (1998) quienes, dentro de las siete características que presenta la información on-line, determinan que una de ellas es la actualización "porque se pueden publicar las noticias en cuanto se producen..." (Edo, 2009.Pàg 3). Poco después, López et al. (2005) concluían, entre otras cosas, que “...la información de última hora importa más que la interactividad” (Edo, 2009. Pàg11). Cristóbal y Marimon (2010), asimismo, concluyen que la posibilidad de contactar con la empresa es uno de los aspectos que más valoran los clientes online, al igual que Wolfinbanger y Gilly (2003) quienes, dentro de los cuatro factores que aislaban como determinantes en cuanto al estudio de la percepción de la calidad, indicaban como tercer factor el "Customer service", donde era importante el hecho de que se pudiera responder al cliente de la forma más rápida posible.

\section{Dimensión c-fiabilidad y privacidad}

\footnotetext{
"Los consumidores online destacan la confidencialidad y la fiabilidad en el servicio prestado... son usuarios preocupados por la seguridad de sus datos y transacciones" (Cristóbal y Marión, 2010. Pág. 109)
}

En el uso de la escala E-S-Qual nos encontramos con autores como Yang y Tsai (2007), Parasuraman et al. (2005) y Vázquez-Casielles et al. (2009), que mantienen que la fiabilidad es uno de los cuatro pilares sobre los que se basa esta nueva escala de medición de los servicios electrónicos. Es más, hay estudios que demuestran como la fiabilidad y la privacidad, de forma conjunta, generan un aumento de la calidad percibida en el website; "la habilidad para realizar el servicio de forma fiable y segura, adaptado a las cualidades del usuario, influye favorablemente en los niveles de calidad de servicio" (Sánchez, 2002. Pág 61). 


\subsection{Identificación del escrito y de la información (c.1)}

Gairín (2001) recoge en su estudio como pregunta clave: ¿Se identifica al autor de la noticia? Donde incluso lo relaciona con otras noticias dentro del mismo portal de información: ¿Es posible acceder a los artículos de un autor concreto? Más recientemente, Rodríguez et al. (2012), en un estudio sobre indicadores para la evaluación de la calidad en los Cibermedios, no incluye la identificación del autor pero sí la "Comunicación con el autor de la noticia" como un indicador de la dimensión que estudia la cooperación con el internauta.

\subsection{Información sobre la empresa (c.2)}

Barnes et al. (2002) ya hacían hincapié en el hecho de que el cliente pudiera contactar con la empresa como un indicador de calidad basándose en el trabajo de Bitner et al. (2000), quienes determinaban que en el uso de tecnologías de la información se debía mantener informado siempre al cliente sobre la empresa con la que trataba. De la misma forma Park y Kim (2003), también concluían que la información sobre el site era un factor a tener en cuenta a la hora de determinar la decisión de compra del consumidor y su posterior lealtad a la página web. Asimismo, Muñoz y González (2008) recalcaban dicha postura.

\subsection{Información sobre el producto y las condiciones de uso (c.3)}

Peterson et al. (2007) recalcaron en un primer momento que, cuanto más extensa y fiable fuera la información disponible sobre el producto en venta on-line, mayor sería la satisfacción del cliente y al mismo tiempo se reduciría su tiempo de búsqueda. También, en unos primeros estudios sobre el comportamiento de compra on-line, Park y Kim (2003) concluían que tanto la información del producto como la del servicio provocaban una mayor satisfacción en el cliente y, además, un aumento en la calidad percibida de la página web y sus servicios. Y no sólo eso, sino que la información sobre el servicio era la más importante de todas.

\subsection{Entrega del producto en el plazo estimado (c.4)}

Según autores como Bhattacherjee (2002) y Pavlou (2003), la reducción de la incertidumbre por parte del cliente a la hora de llevar a cabo ciertas transacciones supone un aumento de la confianza en la transacción electrónica. Del mismo modo, Parasuraman et al. (2005), así como Wolfinbarger y Gilly (2003) también llegan a la conclusión de que existen cuatro factores definitorios de la percepción de la calidad, entre los cuales se encuentran la fiabilidad y privacidad como un todo, indicando que "el producto que el cliente recibe es el que ordenó y en el tiempo prometido" (Wolfinbarger y Gilly, 2003. Pág. 193). De hecho, en un estudio realizado por Marimón y Cristóbal (2012), referente a la distribución en supermercados virtuales, el atributo más valorado fue el de "Entrega los pedidos cuando promete". 
4.5 Protección de datos personales (c.5)

“...los consumidores perciben una mayor competencia y destreza en la organización con la que se relacionan. Por ejemplo, informando a los clientes de la confidencialidad en el tratamiento de sus datos personales y de las plataformas de encriptación de datos utilizadas, que permiten garantizar la seguridad en las transacciones bancarias..." (Lassala et al., 2007. Pág. 41)

Barnes et al. (2002) ya aplicaban dicho indicador en base a los estudios de Wang et al. (1998) sobre la privacidad del consumidor y que podríamos completar con el trabajo de Lwin et al. (2007) a través del cual se indicaba que, ante lugares con baja seguridad en cuanto a protección de datos, se generaba cierta desconfianza por parte del internauta.

\section{Dimensión d-interacción}

"El éxito de la prensa digital depende de su capacidad para crear sitios web que permitan la interacción tanto de los usuarios con los contenidos, como de los usuarios entre sí” (Rodríguez et al., 2010. Pág. 42)

"Una página web que no cuida su diseño e interacción con el internauta proyecta una imagen pobre en Internet y pierde competitividad" (Barnes y Vidgen, 2002. Pág. 114).

\subsection{Participación en los contenidos (d.1)}

Existe un movimiento al que algunos aluden como "Periodismo ciudadano" (Edo, 2009) en el que las personas de a pie de calle aportan noticias, fotos o vídeos que no son incompatibles con la labor periodística de un profesional. Orihuela (2000) los llamaba "prosumidores". Rodríguez y Pedraza (2009) incluyen este tipo de interacción en sus indicadores, y López y Pastor (2010) refuerzan esta base donde el término Envío de noticias propias “....se configura como un servicio atractivo para el usuario ya que con su uso podría participar en la creación de contenidos de portal periodístico, aspecto muy relevante en la sinergia social-informativa actual" (López y Pastor, 2010. Pág. 180)

\subsection{Interacción con la información a través de comentarios (d.2)}

En un estudio comparativo realizado por García de Torres et al. (2008), se destaca el hecho de que los "comentarios en los blogs" y los "comentarios en la noticia" son dos de las siete variables que han evolucionado en los últimos años en cuanto a la importancia de las herramientas 2.0. Hecho al que también hace mención Guallar (2007). Del mismo modo y según el director de The Guardian, Alan Rusbridger, deben trabajar actualmente los ciberperiodistas: incentivando la participación de los 
usuarios y fomentando el debate y la cooperación de los mismos así como sus sugerencias (Salinas, 2012).

\subsection{Personalización de la información (d.3)}

En un principio Pérez y Perea (1998) plantearon, como una de las siete características de la información online, que ésta sea personalizada "porque hace posible la selección activa de los contenidos por parte de los usuarios". Aunque más recientemente, Rodríguez et al. (2012) introducen de nuevo, en los indicadores de su modelo para el análisis de la interacción y de la adopción de la web 2.0, el parámetro 5, personalización de la información: adaptación en función de los contenidos, sindicación de los mismos adaptados a nuevas tecnologías y suscripciones a boletines y sistemas de alertas.

5.4 Sistema de encuestas o mejora o valoración de la noticia o el servicio ofertados (d.4)

\footnotetext{
“...los medios tienen que escuchar y dejar participar más a sus audiencias. Porque esa participación no es algo irrelevante, sino una tendencia creciente que va a conseguir que los medios, convertidos en tantos casos en centros de poder politizados e ideologizados que buscan sus intereses, no tengan más remedio que contar con los lectores y aceptar sugerencias" (Edo, 2009. Pág. 15).
}

Según, Guallar (2007) los diarios digitales renovaron sus páginas web para añadir herramientas 2.0 de interacción, entre las cuales se permitiera un sistema de valoración de la noticia por parte del internauta. Sin embargo, son Díaz y Palacios (2007), quienes en su tratado sobre métodos de investigación en Ciberperiodismo mencionan las encuestas como una metodología complementaria a la hora de analizar los diversos aspectos de un cibermedio. Más recientemente, Rodríguez et al. (2012), en uno de sus indicadores relacionados con la interacción (medio de comunicación-usuario), incluyen el término "votación de noticias publicadas por el medio de comunicación".

\subsection{Información y servicios de pago (d.5)}

Parra et al. (2008) contemplan dicha posibilidad basándose en que "la calidad y la fiabilidad de los contenidos de un ciberperiódico son valores que hay que pagar" (Parra et al., 2008. Pág. 64). Más adelante, García y González (2013) nos detallaban el ejemplo de diversos diarios digitales como Elmundo.es, quiénes usaban diversos servicios de pago de forma rentable con un importante incremento de suscriptores. Ahora bien, concluyen que todavía se están definiendo dichos sistemas y servicios de pago que, pese a ser rentables, no generan excesivos beneficios, al menos no los suficientes para mantener grandes equipos periodísticos. 


\section{Conclusiones}

Así pues, nos encontramos ante una primera aproximación a un modelo de análisis de la calidad percibida en los Cibermedios que intenta arrojar algo de luz sobre una posible (y relativamente nueva) dimensión de estudio que nos pueda facilitar nuevas estrategias de posicionamiento y comunicación, así como las herramientas necesarias para un mejor uso de los portales de información de cara a la aparición de nuevas tecnologías.

Es por ello que, de momento, se proponen estas cuatro dimensiones: eficiencia, disponibilidad del sistema, fiabilidad y privacidad, e interacción desarrolladas a través de 22 ítems, que en un futuro próximo esperamos estudiar mediante los trabajos de campo correspondientes y, de esa manera, poder ofrecer respuesta a cada una de las propuestas así como nuevas vías alternativas para mejorar la relación empresa-cliente a través de los medios de comunicación on-line, independientemente de la plataforma en la que se desarrollen.

\section{Bibliografía}

BARNES, S.J; VIDGEN, R.T. (2002). "An integrative approach to the assessment of e-commerce quality", Journal of Electronic Commerce Research, vol.3, núm.3, p. $114-227$

(2003). "Measuring Web site quality improvements: a case study of the forum on strategic management knowledge exchange". Industrial Management \& Data Systems, vol. 103, núm.5, p. 297-309.

(2006). "Data triangulation and web quality metrics: A case study in e-government". Information \& Management, vol. 43, p. 767-777

BHATTACHERJEE, A. (2002). "Individual Trust in Online Firms: Scale Development and Initial Test", Journal of Management Information Systems, vol. 19, p. 211-241

BITNER, M.J.; OSTROM, A.L.; MEUTER, M.L. (2000). "Implementing successful self-service technologies", Academy of Management Executive, vol.16 (4), p. 96-109

CABRERA GONZÁLEZ, M.A. (2010). Evolución tecnológica y Cibermedios. Editorial Comunicación Social Ediciones y Publicaciones. Salamanca.

CEBRIÁN HERREROS, M. (2009). "Comunicación interactiva en los Cibermedios" Revista Comunicar. Vol. 17, p. 15-24

CODINA, Ll. (2000). "Evaluación de recursos digitales en línea: conceptos, indicadores y métodos". Revista española de Documentación Científica. Vol. 23 núm. 1

COX, J.; DALE, B.G. (2002). "Key Quality Factors in Web Site Design and Use: an Examination", International Journal of Quality and Reliability Management, núm.19(7), p. 864-888 
CRISTÓBAL-FRANSI, E.; MARIMON-VIADIU, F. (2011). "La gestión del supermercado virtual: tipificación del comportamiento del cliente online". Investigaciones Europeas de Dirección y Economía de la Empresa. Vol.17, núm. 1, p. 93-112.

DÍAZ NOCI, J.; SALAVERRÍA, R. (2003). Manual de Redacción Ciberperiodística. Barcelona: Ariel. ISBN 84-3441-129-77

EDO, C. (2009). La noticia y la narración periodística en los Cibermedios y en los entornos comunicativos emergentes. Sala de Prensa, año XI, vol. 5. Disponible en: http://www.saladeprensa.org/art843.htm. Consultado el 17 de abril de 2013.

GARCÍA DE TORRES, E.; RODRÍGUES MARTÍNEZ, J.; SAIZ OLMO, J.; ALBACAR SERRANO, H.; RUIZ GRAU, S.; MARTÍNEZ MARTÍNEZ, S. (2008): La herramientas 2.0 en los diarios españoles 2006-2008: tendencias. Prisma.com. Disponible en: http://revistas.ua.pt/index.php/prismacom/article/viewFile/676/ pdf. Consultado el 3 de febrero de 2013.

GOSENDE, J. (2011). "Cómo la Web 2.0 puede ayudar al posicionamiento en buscadores". Microsoft, Pymes y Autónomos. Disponible en: http://www.microsoft. $\mathrm{com} /$ business/es-es/content/paginas/article.aspx?cbcid=56. Consultado el $20 \mathrm{de}$ mayo de 2013.

GUALLAR, J. (2007). "La renovación de los diarios digitales: rediseños y Web 2.0". El profesional de la información. Vol. 16, núm. 3, p. 235-242.

KAWAMOTO, K. (2003). Digital Journalism. Emerging Media and the Changing Horizons of Journalism. Maryland. Rowman\&Littlefield Publishers.

LASSALA, C.; RUIZ, C.; SANZ, S. (2010). "Implicaciones de la satisfacción, confianza y lealtad". Revista Europea de Dirección y Economía de la Empresa, vol. 19, núm. 1 p. 27-46

LIN, J.; LU, H. (2000). "Towards an Understanding of the Behavioural Intention to Use a Web Site," International Journal of Information Management. Vol. 20, p.197-208

LÓPEZ CARREÑO, R.; PASTOR SÁNCHEZ, J.A. (2010). “Actualización del modelo de portal periodístico de prensa española" Anales de Documentación, vol. 13, p. 177-184.

LWIN, M.; WIRTZ, J.; WILLIAMS, J.D. (2007). "Consumer Online Privacy Concerns and Responses: A Power-Responsbility Equilibrium Perspective." Journal of the Academy of Marketing Science Vol. 35 (4), p. 572-585

MACIÂ, F. (2007). "La Web 2.0: nuevos retos para el posicionamiento en Internet". Human Level Communications. Disponible en: http://www.humanlevel.com/ articulos/web-2-0/la-web-2-0-nuevos-retos-para-el-posicionamiento-en-internet. html. Consultado el 20 de mayo de 2013.

MARIMÓN, F.; CRISTÓBAL, E. (2012). "La influencia de la calidad percibida en el sector de la distribución alimentaria por internet: perspectiva multidimensional aplicada a un supermercado online". Revista de Estudios Empresariales. Segunda época. Núm. 1, p. 131-148.

MASIP, P.; DÍAZ NOCI, J.; DOMINGO, D.; MICÓ SANZ, J.L.; SALAVERRÍA, R. (2010). "Investigación internacional sobre ciberperiodismo: hipertexto, interac- 
tividad, multimedia y convergencia". El profesional de la información, vol. 19, núm. 6, p. 568-576

NIELSEN, J. (2008). Writing Style for Print vs. Web. Alertbox. Disponible en: http:// www.nngroup.com/articles. Consultado el 20 de mayo de 2013.

ORIHUELA, J.L. (2000). Las nuevas tecnologías de la información, claves para el debate. Disponible en: http://www.nuevarevista.net/articulos/las-nuevas-tecnologias-de-la-informacion-claves-para-el-debate. Consultado el 31 de julio de 2013.

PALACIOS, M.; DÍAZ, N. (2009). Online Journalism: Research Methods. A multidisciplinary approach in comparative perspective. Bilbao. Servicio Editorial de la Universidad del País Vasco.

PARASURAMAN, A.; ZEITHAML, Valerie.; MALHOTRA, Alvin (2005). "E-S-QUAL: A Multiple-Item Scale for Assessing eElectronic sService Quality", Journal of Retailing, núm.64 (1), p. 12-40.

PARK, C.; KIM, Y. (2003). "Identifying key factors affecting consumer purchase behavior in an online shopping context", International Journal of Retail \& Distribution Management, Vol. 31, núm. 1, pp.16-29

PARRA, D.; ÁLVAREZ, J. (2004). Ciberperiodismo. Madrid: Síntesis.

PARRA, D.; EDO, C.; FLORES, J.; GARCÍA-ALONSO, PD.; MARCOS, J.C. (2008). "Proceso de transformación de los Cibermedios: los retos de las empresas periodísticas". Revista Latina de Comunicación Social, núm.63, p. 63-70.

PAVLOU, P. (2003). "Consumer Acceptance of Electronic Commerce: Integrating Trust and Risk with the Technology Acceptance Model". International journal of Electronic Commerce. Vol. 7, p. 69-103

PERCEVAL, J.M. (2008). "El estado líquido del ciberperiodismo". Anàlisi: Quaderns de comunicació $i$ cultura, núm.36, p. 79-86

PÉREZ LUQUE, M.J.; PEREA FORONDA, M. (1998). "El reto de crear noticias online. Análisis de la comunicación online actual y perspectivas de futuro", Cuadernos de Documentación Multimedia núm. 6-7, Universidad Complutense, Madrid.

PETERSON, R.A.; BALSUBRAMANIAN, S.; BRONNENBERG, B.J. (2000). "Exploring the implications of the internet for consumer marketing", Journal of the academy of management Science, vol. 25. núm.4, p. 329-46

PETNJI, L.; MARIMON, F.; CASADESÚS, M. (2012). "Assessing e-service quality: the current state of E-S-QUAL", Total Quality Management Business Excellence, núm.23, p. 1363-1378

RODRÍGUEZ, I. (2000). Marketing.com: Marketing y comercio electrónico en la sociedad de la información. Edición Pirámide-ESIC. Madrid.

RODRÍGUEZ-MARTINEZ, R.; CODINA, Ll.; PEDRAZA-JIMÉNEZ, R. (2010). Cibermedios y Web 2.0: Modelo de Análisis, resultados de la aplicación. El profesional de la información. Disponible en: www.lluiscodina.com/periodismo20.pdf. Consultado el 6 de marzo de 2013.

RODRÍGUEZ-MARTÍNEZ, R.; PEDRAZA-JIMÉNEZ, R. (2009). "Prensa digital y Web 2.0"[en linea] Hipertext.net, núm. 7. Disponible en: http://www.upf.edu/ hipertextnet/numero-7/prensa-digital.html. Consultado el 19 de mayo de 2013. 
SALINAS, A. (2012). The Guardian inicia el camino al 'periodismo abierto. UOC. ElPeriódico.com. Disponible en http:/uoc2012.elperiodico.com/2012/06/13/ theguardian-periodismoabierto/

SÁNCHEZ FRANCO, M. (2002). "La calidad percibida del website: un factor de influencia sobre el valor de la marca". Investigación y Marketing núm. 76, p. $55-62$

SCOLARI, C.A.; MICÓ, J.L.; NAVARRO, H.; PARDO, H. (2008). "El periodista polivalente. Transformaciones en el perfil del periodista a partir de la digitalización de los medios audiovisuales catalanes". Zer, vol. 13, núm. 25, p. 37-60

VÁZQUEZ CASIELLES, R.; RÍO LANZA, A.B.; SUÁREZ ÁLVAREZ, L. (2009). "Las agencias de viaje virtuales: ¿Cómo analizar la calidad de e-servicio y sus efectos sobre la satisfacción del cliente?"Universia Business Review, núm.24, p. $122-143$

VILA LÓPEZ, N.; AMOROS KEM, E.; ALDAS MANZAN, J. (2013). "Posicionamiento de marcas políticas a través de la Web". GCG Geortown University-Universia. Vol. 7, núm.1, p. 16-29

VILLAREJO RAMONS, A.F.; SÁNCHEZ FRANCO, M.J. (2004). "La calidad de servicio electrónico: un análisis de los efectos moderadores del comportamiento de uso de la web". Cuadernos de economía y dirección de la empresa, núm. 21, p. $121-125$

WANG, H., LEE, M.K.O., WANG, C. (1998). "Consumer privacy concerns about Internet marketing", Communications of the ACM, Vol. 41, núm.3, p. 63-70.

WOLFINBARGER, M.; GILLY, M. (2003). "eTailQ: Dimensionalizing, Measuring and Predicting etail Quality", Journal of Retailing, Vol. 79, p. 183-198

YANG, H.; TSAI, F. (2007). "General E-S-QUAL Scales Applied To Websites Satisfaction and Loyalty Model". Communications of the IIMA 117, vol. 7, núm. 2, p. 115-126.

\section{Los autores}

Francisco Hernández Soriano. Licenciado en Administración de Empresas por la Universidad de Lleida (UdL) y doctorando en el programa "La construcción Europea" del departamento de Filología Catalana y Comunicación.

Eduard Cristóbal-Fransi. Doctor en Economía por la Universidad de Lleida (UdL). Profesor del área de comercialización e investigación de mercados. Director del departamento de Administración de Empresas en la UdL. Su investigación está centrada en el comercio electrónico, implantación de las TIC y la evaluación de la calidad percibida. Ha participado en congresos nacionales e internacionales y publicado en revistas como International Journal of Market Research, International Journal of Marketing Studies, International Journal of Business and Management, International Journal of Services Technology and Management and Managing Service Quality, y ha colaborado en diferentes libros como "E-Commerce and 
V-Business: Digital Enterprise in the Twenty-First Century" o "Social E-Enterprise: Value Creation through ICT" entre otros. 